\title{
An Artificial Neural Network Approach to Predicting Electrostatic Separation Performance for Food Waste Recovery
}

\author{
Koon Chun Lai*, Soo King Lim, Peh Chiong Teh, Kim Ho Yeap \\ Universiti Tunku Abdul Rahman, Malaysia
}

Received:30 August 2016

Accepted: 13 February 2017

\begin{abstract}
This study presents the empirical exploration of food waste recovery throughout the electrostatic separation process. In addition, the paper discusses the potential of artificial neural network (ANN) in predicting the responses. A five-level three-factor Taguchi orthogonal array (OA) design of experiment was employed as an initiative to optimize the prediction process. The electrostatic separation process was modelled using ANN by considering the recovered food waste and misclassified middling product during separation. A multi-layer feed-forward network developed in MATLAB was constructed. It was found that the results from the experiment and predicted model were in very good agreement. To our best knowledge, this is the first report for prediction of food waste separation performance employing ANN and Taguchi design.
\end{abstract}

Keywords: ANN, Taguchi, waste recovery, separation, electric field

\section{Introduction}

Improper solid waste management could degrade and deteriorate the quality of the environment [1]. Without adequate pre-treatment, disposed waste - especially food waste - at open disposal sites or landfills would cause public nuisances such as air, water, and soil pollution [2]. The massive generation of leachate and landfill gases from the decomposition of food waste may accelerate the pace of environmental degradation and global warming [3]. As an environmentally friendly measure to segregate food waste from municipal solid waste, the roll-type electrostatic separator has been proposed in [4]. Food waste (FW) is detachable from plastic and glass during the

*e-mail: laikc@utar.edu.my rotational separation process owing to the high percentage of moisture level. In other words, FW has relatively higher conductivity than non-FW. With the aid of an electrostatic separator, the FW and non-FW would fall into different locations as desired. The substances are typically charged by friction, induced, or corona charges before being subjected to the electrostatic and gravity forces. During the separation process, however, some of these matters may fall in-between as middling product. The failure would result in decline in separation efficiency. The extent of electrostatic separation could be quantified in terms of food waste recovery and yield of middling product. High recovery with less middling is necessary for an efficient separation process.

Since electrostatic separation segregates substances with different electrical conductivities without involving incineration or chemical treatment, the process is free 
from pollution. It is usually employed in the food security industry to remove hair, ash, or waste straws during packaging [5-6], and in metal recycling plants to recover metals from printed-circuit board waste [7-8]. The performance of the electrostatic separator could be affected by a number of factors. Tripathy et al. reported that temperature and feed rate have influences on both the recovery and grade of the separation process [9], whereas Medles et al. presented the effects of high voltage levels, roll electrode rotating speed, and the splitter angular position [10]. In addition, input factors such as mixture characteristics, particle charge/mass ratio and electrode configurations are found to affect the separating outcomes [11-12]. In short, the literature reveals that electrostatic separation is a complicated process that should be characterized, modeled, and optimized.

An artificial neural network (ANN) is a potential computational tool to be employed to predict separation performance. It has been successfully applied in many problem-solving domains like engineering, accounting, medicine, and geology. This computer model is considered as a system to receive inputs, to modify weights, and train the network before a desired response is produced at the output [13]. ANN can be employed to optimize an existing system or new processes, and to predict the system behaviour with known parameters [1415]. A five-level three-factor Taguchi orthogonal array (OA) design of experiment can be employed as a novel initiative to optimize the predicted results. To the best of our knowledge, this is the first report for prediction of food waste separation performance employing ANN and Taguchi design.

The aim of this study is to develop a model of food waste (FW) separation performance in terms of high FW content recovery and low misclassified middling mass. We investigated the impacts of the key parameters, i.e., power supply, rotation speed, and electrode angle. The effectiveness of this model was then validated by comparing the results obtained from experimental measurements and those predicted using ANN.

\section{Material and Methods}

The test sample, which consisted of a $100 \mathrm{~g}$ mixture of $40 \mathrm{wt} \% \mathrm{FW}$ (fruit peel) and $60 \mathrm{wt} \%$ non-FW (plastic and styrofoam), were first crushed into small pieces and deposited onto the roller of the separator as a monolayer. The water content of the FW ranged from $20 \%$ to $40 \%$. All particles were cut manually into an average size of $3.0 \mathrm{~mm}$ each. An earth-grounded roller type electrostatic separator was employed. An ionizing needle electrode was powered by a DC power source to generate corona discharge. The surrounding air near the ionizing electrode was ionized to form a high intense ionizing zone. An electrostatic plate was connected downstream to provide the non-discharging electrostatic charge. The separated and recovered products were found in the FW, non-FW, and middling collection tanks under the separator.
The separation process relies on a number of forces, e.g., centrifugal force due to the rotation of the roller, lifting force due to the attraction by the electrostatic plate, and gravity force and pinning force due to the ionized effect on the insulative particles [16]. When the roller delivers the particles through the zone, the dissipative FW lose their charge rapidly, keeping them from being pinned for a longer time than the insulative non-FW. With the continuous rotation from the roller, FW particles were subjected to a centrifugal force which is larger than the pinning force. They were then thrown off from the roller into the FW collection tank. The non-FW particles remained pinned to the roller due to the larger pinning force and eventually they fell off into the non-FW tank when the rotation stopped. In order to assess the separation efficiency, the mass of the FW and the misclassified mixture (middling) were collected and measured by a digital precision balance with a resolution of $0.1 \mathrm{~g}$ after each run.

In this study, an ANN model was constructed to predict the recovered food waste $\left(W_{f}\right)$ and middling products $\left(W_{m}\right)$. The Taguchi OA design considers three independent input factors: the voltage level of the DC power supply $(\mathrm{kV})$, the rotation speed of the roller (rpm), and the corona electrode angle (degree) as determined by [16]. The recovered FW and middling mass were selected as the network responses. The experimental parameters and their levels are tabulated in Table 1.

ANN is a numerical estimation method that can be applied to simulate the experimental input variables and determine the governing rules among the corresponding factors [17]. It is a potential preeminent tool to model nonlinear processes such as electrostatic separation with its ability to learn the relationship among independent variables and predict accurate output [18]. There are three stages, namely training, testing, and validation performed by the network. Typically by random sampling method, about $70 \%$ of experimental data is used for training and the rest of the data for testing and validation [19]. The best performance of the ANN architecture is evaluated based on the smallest mean squared error (MSE) and the highest value of coefficient of determination (R-squared), which defines the fit integrity of the experimental data. Both MSE and R-squared are expressed as follows:

$$
\mathrm{MSE}=\sum_{i=1}^{n}\left(t_{i}-y_{i}\right)^{2} / n
$$

Table 1. Factors and their levels.

\begin{tabular}{|c|c|c|c|c|c|c|}
\hline \multirow{2}{*}{ Factor } & \multirow{2}{*}{ Unit } & \multicolumn{5}{|c|}{ Level of factors } \\
\cline { 3 - 7 } & & 1 & 2 & 3 & 4 & 5 \\
\hline Voltage level & $\mathrm{kV}$ & 15 & 20 & 25 & 30 & 35 \\
\hline Rotation speed & $\mathrm{rpm}$ & 45 & 60 & 75 & 90 & 105 \\
\hline Electrode angle & degree & 20 & 30 & 40 & 50 & 60 \\
\hline
\end{tabular}


and

$$
\mathrm{R}^{2}=1-\sum_{i=1}^{n}\left(t_{i}-y_{i}\right)^{2} / \sum_{i=1}^{n}\left(y_{i}\right)^{2}
$$

$\ldots$ where $t$ is the target value, $y$ the output value, and $n$ the number of inputs.

\section{Results and Discussion}

In the present study, the neural network is configured as a multiple input-single output (MISO) model in the MATLAB environment. The architecture of the ANN comprises an input layer, an output layer, and two hidden layers to compute the complex interconnections of neurons intelligently. ANN with two hidden layers would produce better prediction than a single-layer network [20]. The topology of a backpropagation ANN is shown in Fig. 1. It is a 3-10-2-2 structure that has 10 neurons in the first hidden layer and two neurons in the second layer, with constant percentage of training data $(70 \%)$, testing $(15 \%)$, and validation data $(15 \%)$. Nonlinear sigmoid and linear activation functions were used in the hidden layers, respectively. The neurons in the layer are the constitutive units of ANN to receive inputs and sum them to generate an output through a linear activation function. The Levenberg-Marquardt backpropagation training algo-rithm was employed to adjust random initial weights assigned to each of the inputs until satisfactory results were met [21].

In the five-level three-factor Taguchi OA design, 25 sets of experiment were conducted and analyzed. Taguchi OA design reduces the time and cost compared to full factorial design as the latter needs $5^{3}$ or 125 sets of experiment in acquiring the same information [22]. The trained ANN was employed to perform prediction of the output responses. Table 2 tabulates the corresponding experimental data and prediction results. It can be seen that the predicted values are close to the respective empirical values. From
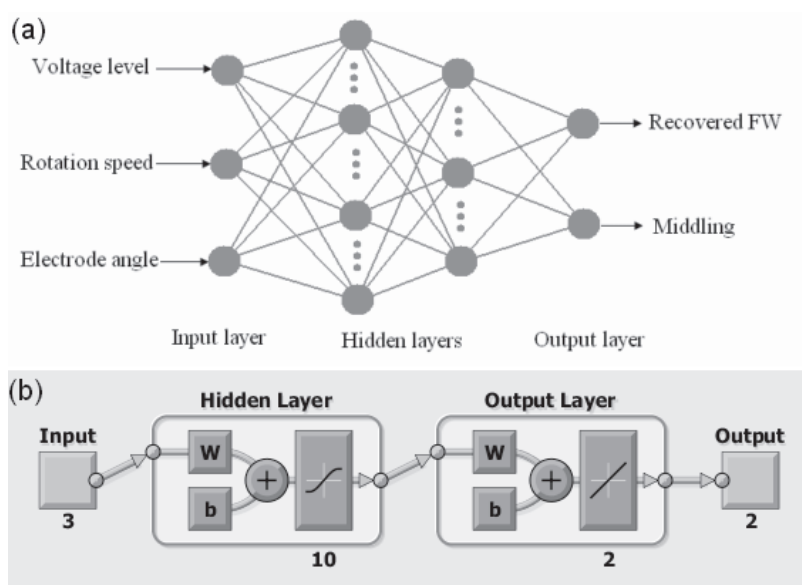

Fig. 1. Topology of the backpropagation ANN in a) schematic view and b) Matlab environment.
Table 2. Experimental data and the predicted results using ANN.

\begin{tabular}{|c|c|c|c|c|}
\hline \multirow{2}{*}{$\begin{array}{c}\text { Exp. } \\
\text { No. }\end{array}$} & \multicolumn{2}{|c|}{$W_{\mathrm{f}}(\mathrm{g})$} & \multicolumn{2}{c|}{$W_{\mathrm{m}}(\mathrm{g})$} \\
\hline & $\begin{array}{c}\text { mental } \\
\text { meri- }\end{array}$ & Predicted & $\begin{array}{c}\text { Experi- } \\
\text { mental }\end{array}$ & Predicted \\
\hline 1 & 10.1 & 10.3 & 65.9 & 66.1 \\
\hline 2 & 18.7 & 18.0 & 38.7 & 41.0 \\
\hline 3 & 24.0 & 24.0 & 23.2 & 23.1 \\
\hline 4 & 24.0 & 24.0 & 25.0 & 24.7 \\
\hline 5 & 18.7 & 19.0 & 43.7 & 42.5 \\
\hline 6 & 21.4 & 19.0 & 38.2 & 48.0 \\
\hline 7 & 26.5 & 26.7 & 21.6 & 21.9 \\
\hline 8 & 25.9 & 26.2 & 22.6 & 22.8 \\
\hline 9 & 19.8 & 20.8 & 40.8 & 39.8 \\
\hline 10 & 10.6 & 10.0 & 67.6 & 65.8 \\
\hline 11 & 29.8 & 29.8 & 19.9 & 20.9 \\
\hline 12 & 28.5 & 28.4 & 20.1 & 21.3 \\
\hline 13 & 21.6 & 22.7 & 37.8 & 36.8 \\
\hline 14 & 10.9 & 10.8 & 66.0 & 66.2 \\
\hline 15 & 20.0 & 19.9 & 40.5 & 40.4 \\
\hline 16 & 32.1 & 32.6 & 17.3 & 14.5 \\
\hline 17 & 24.2 & 25.2 & 34.7 & 33.9 \\
\hline 18 & 11.6 & 11.5 & 64.3 & 64.5 \\
\hline 19 & 20.4 & 20.2 & 37.5 & 38.0 \\
\hline 20 & 25.6 & 25.3 & 22.7 & 23.8 \\
\hline 21 & 27.7 & 28.3 & 31.2 & 29.7 \\
\hline 22 & 12.9 & 12.9 & 62.6 & 62.6 \\
\hline 23 & 21.6 & 21.5 & 34.4 & 34.6 \\
\hline 24 & 26.3 & 25.8 & 18.4 & 22.4 \\
\hline 25 & 25.3 & 26.0 & 20.3 & 22.3 \\
\hline
\end{tabular}

the regression analysis, the R-squared values obtained are 0.988 and 0.979 for $W_{f}$ and $W_{m}$, respectively. This indicates a good prediction for separation performance prediction using ANN.

Network efficiency would be enhanced with optimum percentage of data in the three stages (i.e., training, test, and validation) and the number of hidden neurons. However, these parameters are often determined by trialand-error [23-24]. In order to judge the extrapolating capability, the performances of ANN in relation to the variation in neuron number, testing, and validation dataset were hereby analyzed. Fig. 2 presents the performance of the neural network in terms of MSE and R-squared values due to the variation in the number of neurons. It can be noted that the R-squared value in Fig. 2a) decreases when the number of neurons increases from 10 to 100 . The highest R-squared value was found to be 0.9777 at number 

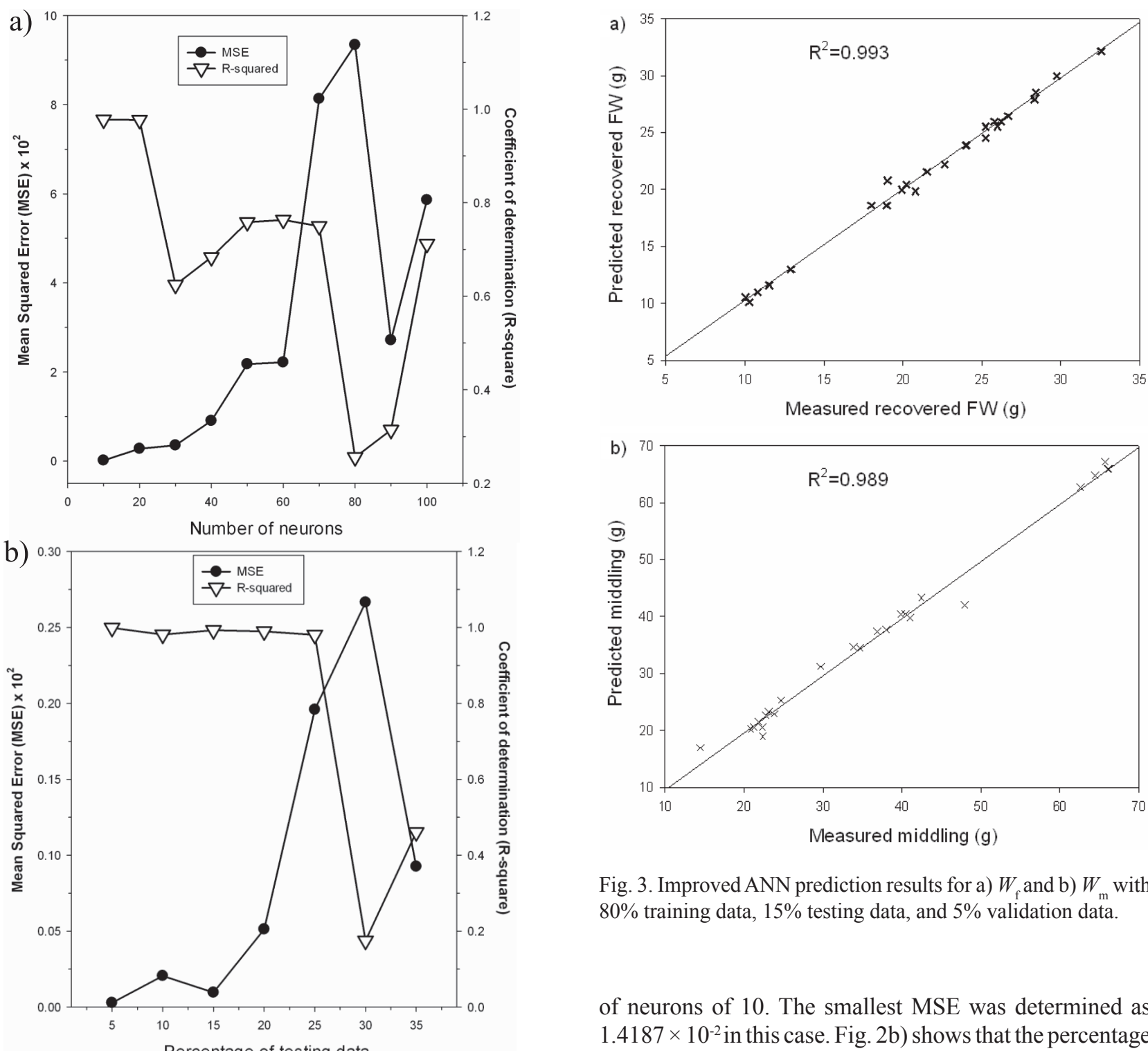

Fig. 3. Improved ANN prediction results for a) $W_{\mathrm{f}}$ and b) $W_{\mathrm{m}}$ with $80 \%$ training data, $15 \%$ testing data, and $5 \%$ validation data.

of neurons of 10 . The smallest MSE was determined as $1.4187 \times 10^{-2}$ in this case. Fig. 2 b) shows that the percentage of testing data varied from $5 \%$ to $35 \%$ so as to evaluate its effect toward network performance. The number of neurons was 10 and the percentage of validation data was set as 5\%. It can be seen that high values of $\mathrm{R}$-squared and small MSE can be achieved at lower number of testing data. Fig. 2c) shows the performance when the percentage of validation data varied from $5 \%$ to $35 \%$, while keeping the percentage of testing data constant at $15 \%$. The maximal R-squared of 0.9922 and the minimal MSE of $2.7326 \times 10^{-3}$ existed when the training data was at $80 \%$ and validation data at 5\%. These findings suggest that a low number of neurons, say 10 , should be selected. Along the same line, the performance of the ANN could be improved with increment of percentage of training data. This approach is useful particularly when the Taguchi design is involved and the set of experimental data is less.

Fig. 3 depicts the ANN predictions with experimental data for recovered $\mathrm{FW}, W_{\mathrm{f}}$ and middling mass, $W_{\mathrm{m}}$. In this case, the number of neurons is 10 with percentage of training data at $80 \%$, testing at $15 \%$ and validation data at 5\%. Higher R-squared values, i.e. 0.993 and 0.989 (compared to the previous 0.988 and 0.979 ) were obtained in both plots, which signify accurate pre- 

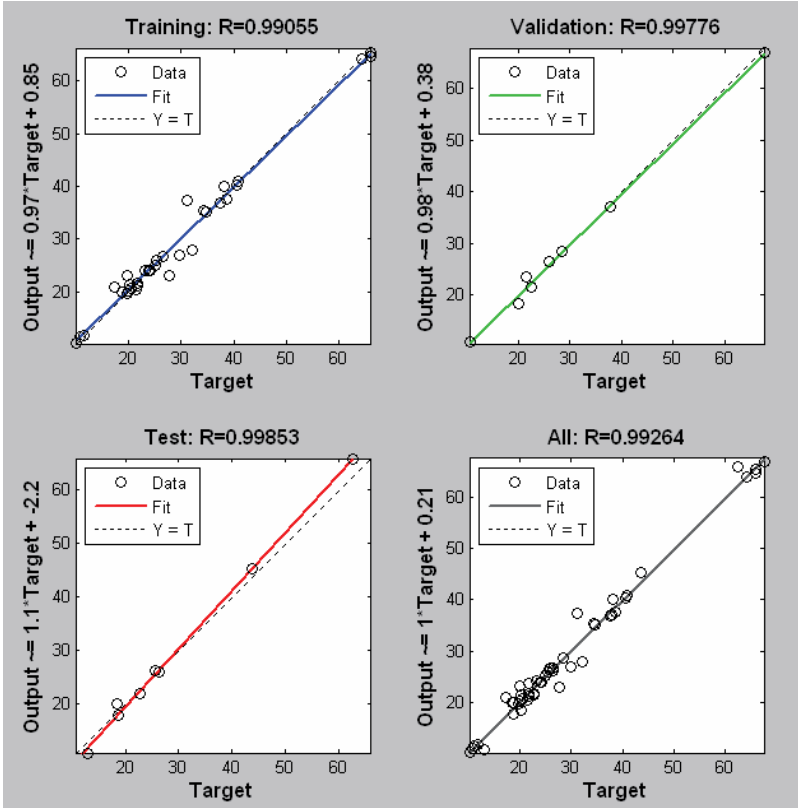

Fig. 4. Parity plots of training, testing, and validation stages.

diction with no more than 0.01 differences between the predicted and target data based on the correlation equations. Fig. 4 presents the R-squared values of 0.9906 , 0.9978, and 0.9985 for training, validation, and test, respectively. It could be summarized that the empirical error is minimized and the results from experiment and predicted model are in excellent agreement.

\section{Conclusion}

In the present study, the modelling of electrostatic separation performance in terms of recovered waste and misclassified middling is designed using ANN based on the Taguchi OA design. The selected input factors are voltage level, rotation speed, and electrode angle as determined in a previous study. The 3-10-2-2 structure of ANN produces good prediction results with R-squared value of about 0.98 , indicating that the presented model is suitable for prediction of the electrostatic separation performance. When a higher percentage of training data is used, from $70 \%$ to $80 \%$ in this study, an improved R-squared of 0.99 is obtained. This implies that performance enhancement of the ANN for prediction can be achieved with low numbers of neuron and adequate training data. The result of the present study can be useful for electrostatic separation process, particularly when the Taguchi design is involved and the set of experimental data is less.

\section{References}

1. BADGIE D., SAMAH M.A.A., MANAF L.A., MUDA, A.B. Assessment of municipal solid waste composition in Malaysia: management, practice and challenges. Pol. J. Environ. Stud. 21(3), 539, 2012.
2. CHRISTENSEN T.H., KJELDSEN P., BJERG P.L., JENSEN D.L., CHRISTENSEN J.B., BAUN A., ALBRECHTSEN H.J., HERON G. Biogeochemistry of landfill leachate plumes. Appl. Geochem. 16, 659, 2001.

3. KHAIRUDDIN N., MANAF L.A., HASSAN M.A., HALIMOON N., AB KARIM W.A.W. Biogas harvesting from organic fraction of municipal solid waste as a renewable energy resource in Malaysia: a review. Pol. J. Environ. Stud. 24 (4), 1477, 2015.

4. LAI K., LIM S., TEH P. Optimization of electrostatic separation process for maximizing biowaste recovery using Taguchi method and ANOVA. Pol. J. Environ. Stud. 24 (3), $1125,2015$.

5. MASUI N. Electrostatic separation for removal from green tea of stems and from food of impurities. Proc. IEJ. 6(3), 159, 1982.

6. MOHANTA S.K., ROUT B., DWARI R.K., REDDY P.S.R., MISHRA B.K. Tribo-electrostatic separation of high ash coking coal washery rejects: Effect of moisture on separation efficiency. Powder Technol. 294, 292, 2016.

7. MOHABUTH N., MILES N. The recovery of recyclable materials from waste electrical and electronic equipment (WEEE) by using vertical vibration separation. Resour. Conserv. Recy. 45, 60, 2005.

8. VEIT H.M., DIEHL T.R., SALAMI A.P., RODRIGUES J.S., BERNARDES A.M., TENÓRIO J.A.S. Utilization of magnetic and electrostatic separation in the recycling of printed circuit boards scrap. Waste Manage. 25 (1), 67, 2005.

9. TRIPATHY S.K., RAMAMURTHY Y., KUMAR C.R. Modeling of high-tension roll separator for separation of titanium bearing minerals. Powder Technol. 201 (2), 181, 2010.

10. MEDLES K., DASCALESCU L., TILMATINE A., BENDAOUD A., YOUNES M. Experimental modeling of the electrostatic separation of granular materials. Particul. Sci. Technol. 25 (2), 163, 2007.

11. BILICI M., DASCALESCU L., BARNA V., GYORGY T., RAHOU F., SAMUILA A. Experimental modeling of the tribo-aero-electrostatic separation of mixed granular plastics. IEEE Industry Applications Society Annual Meeting, 2011.

12. DASCALESCU L., TILMATINE A., AMAN F., MIHAILESCU M. Optimization of electrostatic separation processes using response surface modeling. IEEE T. Ind. Appl. 40 (1), 53, 2004.

13. FARIS H., ALKASASSBEH M., RODAN A. Artificial neural networks for surface ozone prediction: Models and analysis. Pol. J. Environ. Stud. 23 (2), 341, 2014.

14. SAMLI R., SIVRI N., SEVGEN S., KIREMITCI V.Z. Applying artificial neural networks for the estimation of chlorophyll-a concentrations along the Istanbul coast. Pol. J. Environ. Stud. 23 (4), 1281, 2014.

15. SINHA K., CHOWDHURY S., SAHA P.D., DATTA, S. Modeling of microwave-assisted extraction of natural dye from seeds of Bixa orellana (Annatto) using response surface methodology (RSM) and artificial neural network (ANN). Ind. Crops Prod. 41, 165, 2013.

16. LAI K.C., LIM S.K., TEH P.C., YEAP K.H. Characterization of novel food waste recovery process using an electrostatic separator. Pol. J. Environ. Stud. 25 (5), 2227, 2016.

17. CHAIREZ I., GARCÍA-PEÑA I., CABRERA A. Dynamic numerical reconstruction of a fungal biofiltration system using differential neural network. J. Process Control 19 (7), 1103, 2009.

18. BASHEER IA., HAJMEER M. Artificial neural network: fundamentals, computing, design, and application. J. Microbiol. Meth. 43 (1), 3, 2000. 
19. ESFANDIAN H., SAMADI-MAYBODI A., PARVINI M., KHOSHANDAM B. Development of a novel method for the removal of diazinon pesticide from aqueous solution and modeling by artificial neural networks (ANN). J. Ind. Eng. Chem. 35, 295, 2016.

20. ZAFARI A., KIANMEHR M.H., ABDOLAHZADEH R. Modeling the effect of extrusion parameters on density of biomass pellet using artificial neural network. Int. J. Recycl. Org. Waste Agric. 2 (1), 1, 2013.

21. DEMIR S., KARADENIZ A., DEMIR N.M. Using steepness coefficient to improve artificial neural network performance for environmental modeling. Pol. J. Environ. Stud. 25 (4), 1467, 2016.
22. DAVID, C., ARIVAZHAGAN, M., IBRAHIM, M. Spent wash decolourization using nano $-\mathrm{Al}_{2} \mathrm{O}_{3} /$ kaolin photocatalyst: Taguchi and ANN approach. J Saudi Chem. Soc. 19, 537, 2015.

23. PODSTAWCZYK D., WITEK-KROWIAK A., DAWIEC A., BHATNAGAR, A. Biosorption of copper (II) ions by flax meal: empirical modeling and process optimization by response surface methodology (RSM) and artificial neural network (ANN) simulation. Ecol. Eng. 83, 364, 2015.

24. SOLTANALI S., HALLADJ R., TAYYEBI S., RASHIDI A. Neural network and genetic algorithm for modeling and optimization of effective parameters on synthesized ZSM-5 particle size. Mater. Lett. 136, 138, 2014. 